\title{
Factors Associated with Hyponatremia in Patients Newly Prescribed Citalopram: A Retrospective Observational Study
}

\author{
Andrea Christine Shysh ${ }^{1,2,3} \cdot$ Zahinoor Ismail $^{1,4,5,6,7} \cdot$ Davinder Sidhu $^{1,3} \cdot$ Maggie Guo $^{3} \cdot$ Leonard T. Nguyen $^{1,3}$. \\ Christopher Naugler ${ }^{1,2,3,5}$
}

Accepted: 29 April 2021 / Published online: 23 May 2021

(c) The Author(s) 2021

\begin{abstract}
Background Hyponatremia is a common and under-recognized adverse drug reaction of selective serotonin re-uptake inhibitor (SSRI) antidepressants. Despite its clinical importance, there are few large-scale studies on the factors associated with hyponatremia.

Objective The aim of this study was to determine the incidence of hyponatremia and to identify patient factors associated with hyponatremia in a large, population-based cohort initiating new prescriptions for citalopram.

Methods We included all patients with a new prescription for citalopram during 2010-2017, inclusive, with baseline and post-initiation serum sodium values available. Data were obtained from an Alberta Health Pharmacy database to identify new citalopram prescriptions. Laboratory values for patients with new prescriptions were obtained from linked Calgary Laboratory Services data. Incident hyponatremia was defined as serum sodium level $<135 \mathrm{mmol} / \mathrm{L}$, following prescription initiation. Associations were determined by performing Cox regression with time-varying covariate analysis, with the development of hyponatremia as the dependent variable.

Results A total of 19,679 patients with new prescriptions were identified; 12,842 females and 6837 males. The mean age was 55.48 years (SD 21.35). Of these patients, 3250 (16.5\%) developed hyponatremia, 1996 (15.5\% of) females and 1254 $(18.3 \%$ of $)$ males $(p=0.002)$. Cox regression showed significant associations of hyponatremia with lower baseline sodium (HR 0.788), older age (HR 1.029), thiazide diuretic use (HR 1.141), and male sex (HR 1.168). Pharmaceutical manufacturer or strength of citalopram did not have significant effects on the development of hyponatremia.

Conclusion This study provides additional data on the predictors of hyponatremia among patients initiating citalopram therapy. We report a $16.5 \%$ incidence of hyponatremia after starting citalopram treatment, and significant new findings include a higher incidence in males. This is the first published incidence of hyponatremia following the initiation of citalopram treatment across all ages in Canada.
\end{abstract}

\section{Key Points}

Hyponatremia is a common side effect of citalopram treatment.

Male sex, lower baseline sodium, concomitant treatment with thiazide diuretics, and older age were independent predictors for patients developing hyponatremia while on treatment with citalopram.

Christopher Naugler

ctnaugle@ucalgary.ca

Extended author information available on the last page of the article

\section{Introduction}

The global increase in antidepressant use over the last two decades has been attributed to the rise in selective serotonin re-uptake inhibitor (SSRI) antidepressants, of which citalopram is the most frequently used [1-4]. Antidepressant use increased over all age groups from the 1990s to the 2000s in the United States, United Kingdom, and the Netherlands [5-7]. In each case, the increase was mainly due to the rise in SSRI use, particularly among the elderly [4, 7]. Despite the global increase in antidepressant use, the frequency of antidepressant treatment in Canada has stabilized over the last 5 years [8], with the exception of citalopram, which quadrupled in use [2]. Additionally, citalopram is the most common SSRI used 
in Sweden, Denmark, and in American nursing homes [1, $3,6]$. The dominance of citalopram over other SSRIs and non-SSRI antidepressants is presumably a result of fewer drug interactions and side effects [9], although its use has been implicated in QT interval prolongation and hyponatremia $[10,11]$.

Hyponatremia occurs as a result of disease or adverse drug reactions and impacts patient health and healthcare systems. Hyponatremia is an electrolyte deficiency defined as serum sodium concentration $<135 \mathrm{mmol} / \mathrm{L}$. While a reduction in serum sodium can arise from preexisting medical conditions, most notably congestive heart failure and chronic kidney disease, hyponatremia is also a common side effect of SSRI antidepressants. The development of hyponatremia in hospitalized patients results in increased mortality, correlated with the severity of plasma sodium depletion [12]. The symptoms of mild to moderate hyponatremia, $<132 \mathrm{mmol} / \mathrm{L}$ and $<128 \mathrm{mmol} / \mathrm{L}$ serum sodium, respectively, include nausea, fatigue, confusion, cramping, unsteadiness, dizziness, and depressed reflexes while severe hyponatremia, $<125 \mathrm{mmol} / \mathrm{L}$ serum sodium, can result in delirium, seizures, coma, and death [13]. The clinical consequences of hyponatremia include increased incidence of hospitalization, longer length of hospital stay, and larger financial burden on the healthcare system [13].

Despite the fact that several previous studies have looked into the incidence and risk factors related to hyponatremia with SSRI use, results have been mixed and very rarely have findings for individual SSRI drugs been reported [14-18]. These studies reported that the risk of hyponatremia was greater in females and also increased with age and thiazide diuretic use [13]. Naturally, those with lower baseline serum sodium levels were at a higher risk for hyponatremia on citalopram treatment as well [13]. Symptoms of renal, cardiac, or metabolic disease, such as hypertension, hypothyroidism, or the use of calcium channel blockers or other, non-thiazide diuretics, have also been significantly associated with hyponatremia [13]. A handful of retrospective, population-based cohort studies assessing antidepressant-induced hyponatremia reinforce the trends seen in the case studies, but are either partially or wholly based on ICD-10 codes instead of actual laboratory data, characterize instances of hyponatremia between different antidepressants, and tend to focus on adults over the age of 65 years instead of the whole population [1, 3, $13,19,20]$.

The goal of this study was to estimate the incidence of hyponatremia and to identify risk factors in a large, population-based cohort with new prescriptions for citalopram. This study is based on population level data from Calgary, Alberta from 2010 to 2017. This region is serviced by a single laboratory, thereby allowing analysis of the entire population taking citalopram within the Calgary Metropolitan Area (population of approximately 1.3 million residents).

\section{Study Design}

\subsection{Source of Data}

Data were obtained from the Alberta Health Pharmacy Information Network (PIN) database to identify all new citalopram prescriptions from 2010 to 2017, inclusive. Date of medication initiation, manufacturer, and dose were recorded. A handful of databases linked by a unique patient identifier, the Alberta Provincial Health Number (PHN), were used to generate patient demographics and collect diagnostic laboratory data. The PIN database, part of the Alberta Netcare Electronic Health Record system, provided access to patient medication profiles and basic demographic information. The PHN was matched to the patient's postal code using a Provincial registry to ensure that only patients from the Calgary Metropolitan Area were included. Lastly, the Calgary Laboratory Services laboratory information system provided diagnostic data on blood tests collected within the established time frame. Calgary Laboratory Services is a regionalized laboratory providing diagnostic services to Calgary and the surrounding area in Southern Alberta.

\subsection{Case Inclusion}

Inclusion of patient data was restricted to those residing in the Calgary Metropolitan Area with a new prescription for citalopram, regardless of strength, dosage, or Drug Identification Number (DIN) from 2010 to 2017. A new prescription was defined as no previous antidepressant treatment 6 months prior to citalopram initiation. Patients with new prescriptions were linked with Calgary Laboratory Services data to identify those who developed hyponatremia following citalopram treatment. Hyponatremia was defined as a serum sodium level $<135 \mathrm{mmol} / \mathrm{L}$. A baseline serum sodium concentration, to a maximum of 6 months pre-treatment, was required. The baseline values were compared with serum laboratory data collected up to 365 days after the start of citalopram treatment. The first instance of a serum sodium level $<135 \mathrm{mmol} / \mathrm{L}$ was considered a hyponatremic event in cases with multiple, sequential serum laboratory collections.

\subsection{Statistical Analyses}

Summary statistics were performed with Chi-squared analysis to measure baseline characteristics, such as means and proportions. Associations were then determined by performing Cox regression with time-varying covariates with the development of hyponatremia as the dependent variable. 
Sex, age, baseline sodium, pharmaceutical manufacturer, citalopram strength, and thiazide diuretic use were the covariates examined. Thiazide diuretic use was defined as treatment with thiazide diuretics within 6 months before starting the citalopram prescription. All data analyses and calculations were done in R Studio [21] using R version 3.5.1 [22]. Adjusted hazard ratios are reported.

\section{Results}

We identified 19,679 patients with new citalopram prescriptions from 2010 to 2017 who met the inclusion criteria for this study, out of a total of 220,946 total patients with at least one prescription for citalopram in our database (Fig. 1). The baseline characteristics of the study population are described in Table 1. Citalopram users were predominantly female, with an average age (standard deviation) of 54.63 (21.94) years. Thiazide diuretic use was documented in 2327 cases (11.8\%), and the average age of diuretic users was 69.38 (14.33) years, compared with that of non-diuretic users, which was 53.61 (21.45) years old. Baseline sodium measurements were similar between females and males, and were also similar between diuretic and non-diuretic users, 138.78 (3.70) $\mathrm{mmol} / \mathrm{L}$ and 139.52 (3.07) $\mathrm{mmol} / \mathrm{L}$, respectively.

Of patients with new prescriptions for citalopram, 3250 (16.5\%) developed hyponatremia: 1996 (15.5\% of) females and $1254(18.3 \%$ of $)$ males $(p=0.002)$. The average age of those who developed hyponatremia was 67.75 (18.74) years, greater than the average age of 53.05 (20.99) years in those who did not $(p<0.001)$. Of patients on concurrent thiazide diuretic use, $662(28.4 \%)$ developed hyponatremia, compared with 2588 (14.9\%) patients who developed hyponatremia but were not using diuretic medications $(p<0.001)$. The mean baseline sodium level was $136.76(4.16) \mathrm{mmol} / \mathrm{L}$ in those that developed hyponatremia, lower than the baseline sodium levels for those who did not develop hyponatremia for which the mean was 139.96 (2.62) mmol/L $(p<0.001)$.

Medications were prescribed from 17 different manufacturers (trade name Celexa and 16 generic formulations). Two manufacturers (Manufacturer E and Manufacturer J) had statistically significant increased hazard ratios for the development of hyponatremia. However, the number of patients prescribed these formulations were small and so this finding must be interpreted with caution.

The 20-mg strength of citalopram was the most common strength prescribed and was dispensed to more than 13,000 patients. Fewer than 6000 patients were prescribed citalopram $10 \mathrm{mg}$, with the 40-mg and 30-mg strengths only treating $<700$ and $<100$ patients, respectively. Since so few individuals were treated with the $30-\mathrm{mg}$ strength, these patients

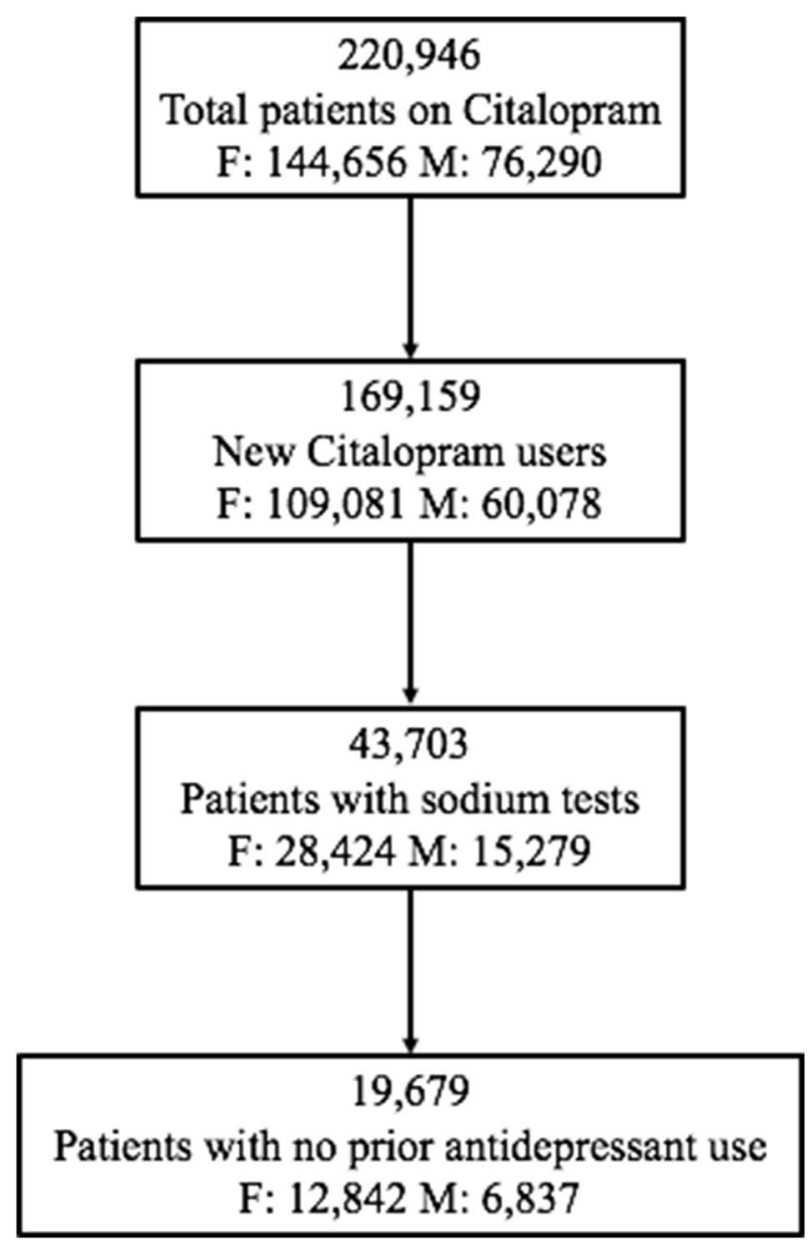

Fig. 1 Case selection. Cases were limited to residents of the Calgary Metropolitan Area from 2010 to 2017, inclusive

were grouped together with those on citalopram $40 \mathrm{mg}$ for subsequent analyses. Almost $20 \%$ of patients on citalopram $10 \mathrm{mg}$ developed hyponatremia; however, this result was not statistically significant $(p>0.05)$.

Results from the risk factor analysis are summarized in Table 2, with a total cumulative hazard risk shown in Fig. 2. Baseline sodium and age violated the proportional hazard assumption and were corrected to satisfy the global goodness-of-fit. Sex, diuretic use, age, and baseline sodium were all significant risk factors for the development of hyponatremia $(p<0.05)$, further illustrated in the respective hazard curves (Figs. 3, 4, 5, 6). Males and concurrent diuretic use had hazard ratios of 1.168 [95\% CI 1.088-1.254] and 1.141 [95\% CI 1.045-1.246], respectively. Age had a hazard ratio of 1.029 [95\% CI 1.027-1.032] for each increasing year of age for the development of hyponatremia, and baseline sodium level (mmol/L) had a hazard ratio of 0.788 [95\% CI $0.780-0.796]$. The strength of citalopram was not a significant risk factor for hyponatremia events $(p>0.05)$. There 
Table 1 Baseline characteristics of patients on citalopram treatment

\begin{tabular}{llll}
\hline & Sex & & Total $(n=19,679)$ \\
\cline { 2 - 4 } & Female $(n=12,842)$ & Male $(n=6837)$ & \\
\hline Age (years) & $54.63(21.94)$ & $57.08(20.10)$ & $55.48(21.35)$ \\
Thiazide diuretic use (\%) & 12.3 & 10.9 & 11.8 \\
Baseline sodium (mmol/L) & $139.39(3.15)$ & $139.52(3.19)$ & $139.43(3.16)$ \\
\hline
\end{tabular}

Age and baseline sodium are represented as mean (SD), and thiazide diuretic use is expressed as a percentage of the population

\begin{tabular}{lccr}
\hline Covariate & $\begin{array}{l}\text { Adjusted hazard } \\
\text { ratio }\end{array}$ & $95 \%$ Confidence interval & $p$ value \\
\hline Sex (male) & 1.168 & $1.088-1.254$ & $<\mathbf{0 . 0 0 0}$ \\
Age & 1.029 & $1.027-1.032$ & $<\mathbf{0 . 0 0 0}$ \\
Diuretic use & 1.141 & $1.045-1.246$ & $\mathbf{0 . 0 0 3}$ \\
Baseline sodium & 0.788 & $0.780-0.796$ & $<\mathbf{0 . 0 0 0}$ \\
Strength & & & \\
20 mg & 0.929 & $0.835-1.034$ & 0.180 \\
40 mg & 1.056 & $0.853-1.306$ & 0.619 \\
Manufacturer & & & \\
Generic Manufacturer A & 1.063 & $0.900-1.256$ & 0.469 \\
Generic Manufacturer B & 1.083 & $0.951-1.233$ & 0.232 \\
Generic Manufacturer C & 1.028 & $0.893-1.183$ & 0.702 \\
Brand name Celexa & 0.839 & $0.634-1.110$ & 0.219 \\
Generic Manufacturer D & 1.924 & $0.959-3.360$ & 0.065 \\
Generic Manufacturer E & 1.524 & $1.094-2.122$ & $\mathbf{0 . 0 1 3}$ \\
Generic Manufacturer F & 0.781 & $0.292-2.088$ & 0.623 \\
Generic Manufacturer G & 0.949 & $0.827-1.090$ & 0.460 \\
Generic Manufacturer H & 1.133 & $0.905-1.418$ & 0.278 \\
Generic Manufacturer I & 0.653 & $0.271-1.572$ & 0.342 \\
Generic Manufacturer J & 1.303 & $1.043-1.629$ & $\mathbf{0 . 0 2 0}$ \\
Generic Manufacturer K & 1.025 & $0.734-1.431$ & 0.886 \\
Generic Manufacturer L & 0.995 & $0.862-1.148$ & 0.941 \\
Generic Manufacturer M & $<0.000$ & $<0.000->9999$ & 0.970 \\
Generic Manufacturer N & 1.138 & $0.847-1.529$ & 0.390 \\
Generic Manufacturer O & 0.875 & $0.866-1.336$ & 0.664 \\
Generic Manufacturer P & 1.076 & & 0.509 \\
\hline & & &
\end{tabular}

Bolded $p$ values indicate significance
Table 2 Variables associated with the development of hyponatremia in patients starting a new citalopram prescription were no significant differences in hyponatremia risk among different manufacturers.

\section{Discussion}

This study is the first to provide population-based incidence data and risk factor analysis for the development of hyponatremia using serum laboratory data, following the initiation of citalopram treatment, inclusive of all ages. The reported incidence of hyponatremia after SSRI use ranged between $0.06 \%$ and $40 \%$ in existing literature; however, this incidence range was based on case reports and case-controlled studies of $<1000$ individuals [23]. Additionally, older case studies defined hyponatremia as a serum sodium level $<130$ $\mathrm{mmol} / \mathrm{L}$, while more recent studies use serum sodium levels of $135 \mathrm{mmol} / \mathrm{L}$ as the cutoff diagnostic value [23-28]. Repeating the analysis using $130 \mathrm{mmol} / \mathrm{L}$ as the definition of hyponatremia resulted in an incidence of $4.8 \%$, with only age and baseline sodium as significant risk factors.

The significance of age as a risk factor for the development of hyponatremia is a well-documented outcome, based on the increased likelihood of physiological deficits and disease with age. Existing studies are predominantly focused on 


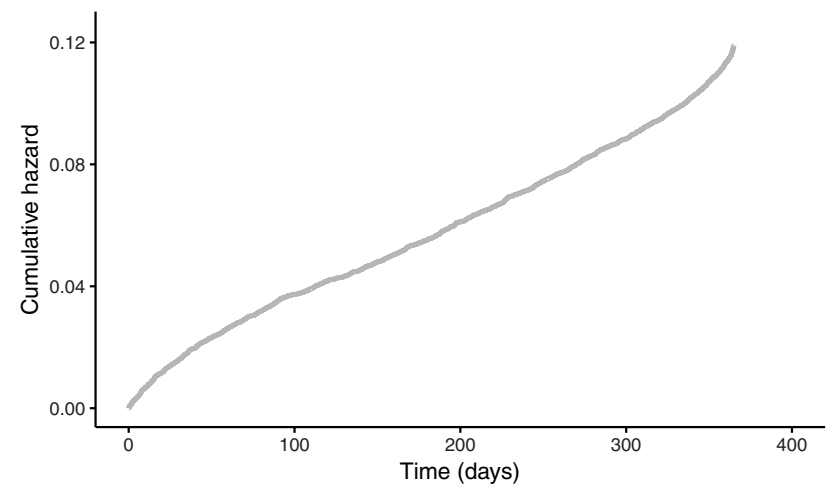

Fig. 2 The total cumulative hazard of hyponatremia following citalopram treatment over time

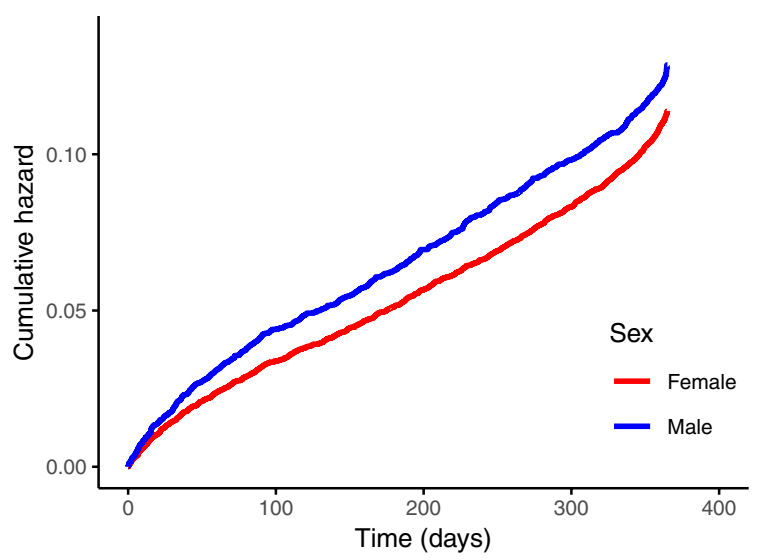

Fig. 3 The cumulative hazard of hyponatremia following citalopram treatment over time, stratified by sex

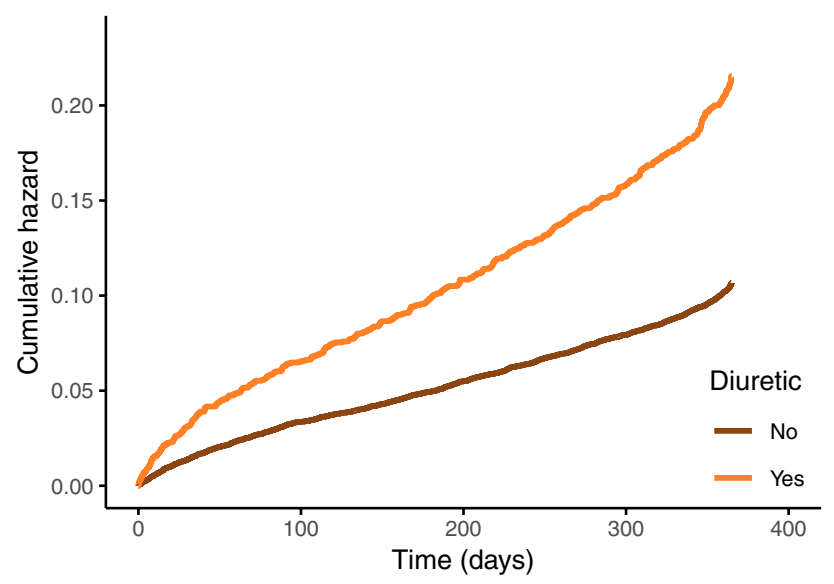

Fig. 4 The cumulative hazard of hyponatremia following citalopram treatment over time, stratified by diuretic use.

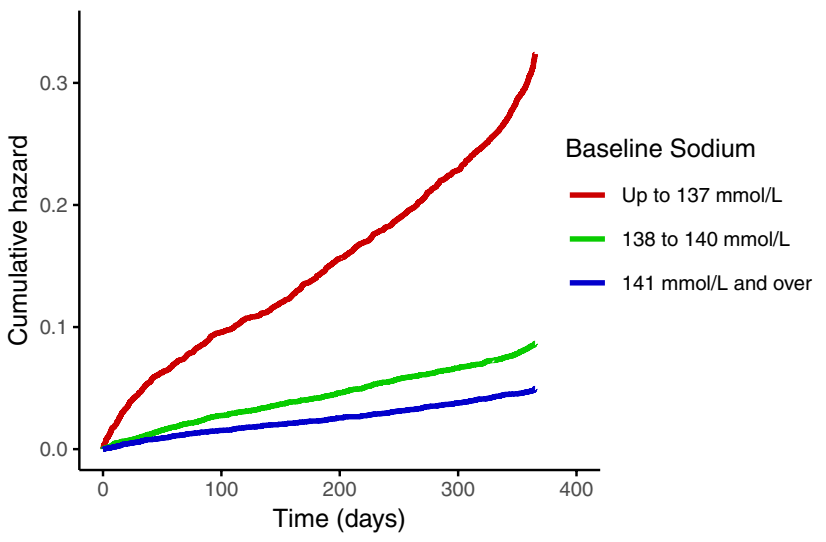

Fig. 5 The cumulative hazard of hyponatremia following citalopram treatment over time, stratified by baseline sodium categories $(\mathrm{mmol} / \mathrm{L})$

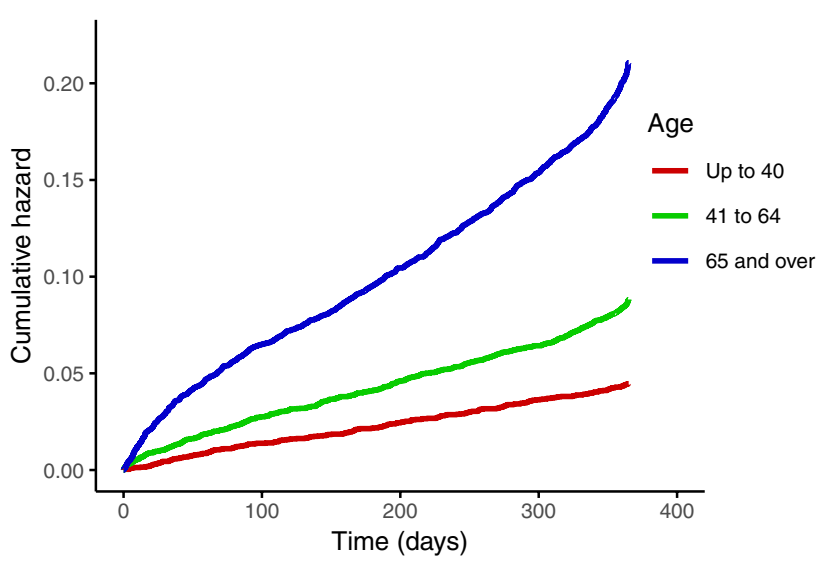

Fig. 6 The cumulative hazard of hyponatremia following citalopram treatment over time, stratified by age (years)

elderly populations $[13,19,20,24,29,30]$, since hyponatremia is more prevalent in aging populations $[13,31]$. Older individuals tend to have more comorbid conditions and are generally treated with a higher number of prescriptions, which may favor the event of an adverse drug reaction $[13,30]$. Physiological changes associated with age, such as kidney volume loss and a decreased ability to regulate electrolyte concentration, result in a reduced ability to cope with the stress of drug-induced hyponatremia [13, 30, 32]. It is important to note that the risk of hyponatremia may not be equal in all elderly patients, with possible influence from genetic polymorphisms in the cytochrome $\mathrm{P} 450$ enzymes [28].

Unsurprisingly, lower baseline serum sodium levels were associated with a greater risk of hyponatremia since a smaller reduction in serum sodium was required to reach hyponatremic levels [32]. Although hyponatremia is the most common electrolyte disorder, with an even higher 
prevalence in older and hospitalized groups [31], it is often under-reported [33]. The clinical features of low serum sodium are often nonspecific, ranging from nausea and cramping to confusion and delirium, and can easily be mistaken for symptoms of the underlying illness [13]. However, even pre-existing, asymptomatic hyponatremia is associated with changes in mental status and mortality, especially in the elderly population [32], and the long-term risks of undiagnosed, asymptomatic hyponatremia are unknown.

Simultaneous thiazide diuretic use with citalopram treatment was another expected risk factor in the development of hyponatremia, since the pathway of thiazide-induced hyponatremia is well understood. Diuretic use, even without concurrent citalopram treatment, has a reported incidence of hyponatremia in the range of $4-14 \%$, with risk factors including age and female sex as well [34]. Thiazide-induced increases in fluid ingestion and impaired water excretion, mediated by antidiuretic hormone (ADH) or urea mechanisms, are known to result in hyponatremia [34]. Although the exact mechanisms of SSRI-stimulated hyponatremia are not confirmed, it is thought that serotonin mediation elevates the concentration of circulating $\mathrm{ADH}$ to increase the reabsorption of water, regardless of declining sodium levels $[1,12,13]$. In terms of hyponatremia, a synergistic effect hinging on inappropriate ADH release may exist between thiazide diuretics and SSRIs [35].

The increased risk of hyponatremia on citalopram in males, in comparison with females, has not been previously reported. Although some studies did not find an association between sex and the risk of hyponatremia, the majority of research identified female sex as a significant risk factor [19, 23-28]. However, these findings were either based on a review of case reports or small, case-controlled studies, and thus had a limited number of patients eligible for inclusion in the analysis. Since the majority of citalopram users were elderly and female, the instances of hyponatremia in the smaller studies may inadvertently bias the risk analysis reviews. The greater prevalence of female antidepressant use compared with that of men is possibly due to a greater willingness for women to perceive and explore treatment for depression from their physicians, or the longer life expectancy of females [36], in addition to the well-established higher prevalence of depression in women [37]. The large patient population included in this study, coupled with robust statistical analysis, circumvented the problem of inclusion bias present in analyzing case reports and smaller case-control studies and may more accurately reflect the increased risk of hyponatremia in males.

The mechanism behind the difference in risk between sexes is unknown, but likely related to the still-uncertain mechanism of citalopram-induced hyponatremia. The average serum sodium level is lower in females than in men, except for those aged 50-70 years [38], and potential differences in intracellular sodium pathways and metabolism is thought to increase the risk of hyponatremia for females [13]. Sexual dimorphism in renal transporters were reported in mice and resulted in differences in water and electrolyte homeostasis [39]. Females also tend to have lower body weight, which is another published risk factor for the development of hyponatremia on SSRI treatment [23, 24, 28]. Since males experience greater serotonin reuptake transporter binding than depressed women [40], it is possible that differences in the serotonin pathway itself may contribute to the increased burden of hyponatremia seen in males. Nevertheless, further insight into the pathway(s) responsible for drug-induced hyponatremia may yield information on the differences seen between sexes.

Two manufacturers showed modestly increased hazard ratios, although this finding should be interpreted with caution as the manufacturers in question were responsible for a small number of prescriptions. Moreover, the manufacturer results varied depending on which pharmaceutical company was chosen as the reference group, suggesting a more complicated relationship between generic and brand citalopram forms. Although all forms of citalopram are considered bioequivalent at each respective strength, the Food and Drug Administration accepts a bioavailability range of $80-125 \%$ compared with the original Celexa formula [41]. Thus, further research is required into this interesting finding. Additionally, variations between product batches have been reported in cases of adverse drug reactions [41], although it is challenging to assess batch differences in large population surveillance studies.

The dose of citalopram treatment did not have significant effects on the risk of developing hyponatremia, which may be a result of the wide therapeutic range of citalopram and clinical dose titration. The therapeutic efficacy of citalopram ranges from 10 to $60 \mathrm{mg} / \mathrm{day}$ [42]. Since plasma serotonin concentrations are significantly decreased at 4 and 8 weeks of therapy [43], any therapeutic and adverse effects are likely to occur in that time. Dose titration is not essential for SSRI use; however, clinicians may choose to gradually increase the dose to effective levels in order to reduce the incidence of adverse reactions [42]. Finally, pharmacokinetic and pharmacodynamics differences at the individual level can also contribute to altered drug exposure, and potentially differential adverse drug reaction profiles [44]. As this type of data is not collected in clinical samples, the role of these differences is speculative.

Given the high incidence rate of hyponatremia provided by this study, and the low percentage of patients with regular serum sodium values available, select patients should receive targeted sodium testing. The cost of serum sodium testing has a reference median cost of $\$ 5 \mathrm{CAD}$, as part of an electrolyte panel, and it is one of the most inappropriately repeated laboratory tests in the region [45]. While it is 
not cost effective to regularly test serum sodium levels for every new citalopram user, select patients at risk for developing hyponatremia on citalopram treatment should receive sodium testing. Older adults, individuals with established lower baseline sodium levels, and those on concurrent thiazide diuretic use should receive sodium testing before and within the first 14 days of citalopram treatment. Although the majority of hyponatremia instances have been reported within the first 2 weeks of treatment [1], clinical judgement should be exercised for the sodium testing schedule, especially if the patient experiences symptoms of hyponatremia.

A selection bias in this study exists since the incidence and risk factor analysis was based on the patients that complete more frequent diagnostic laboratory testing. Overall, females represent the majority of the tested population in Calgary [46]. If an increased rate of testing in males had been observed, it may have offered an explanation for the higher incidence of hyponatremia compared with that of females. However, the proportion of patients with sodium tests within the new citalopram user population was about $25 \%$ for both males and females (Fig. 1). The frequency of diagnostic laboratory testing increases with age, since the likelihood of comorbidity and polypharmacy also increase with age. Older patients tend to have more contact with their healthcare providers, and thus are more likely to complete laboratory testing than those younger in age [47]. Polypharmacy further increases the risk of adverse drug interactions; therefore, patients on high-risk medications, and also those on thiazide diuretics, are significantly more likely to complete regular testing as well $[47,48]$. However, new medication users are less likely to complete ordered tests, and a study on elderly hypertensive patients without comorbidities revealed that two-thirds of the patients had no serum electrolyte measurements before or after therapy initiation [48]. Since the older patients, especially those on thiazide diuretics, were more likely to complete regular laboratory testing, the incidence rate of hyponatremia may overestimate the true occurrence. However, since only $25 \%$ of the total number of new citalopram users in our study had baseline and follow-up sodium tests (Fig. 1), the instance of hyponatremia may have been missed in the majority of patients.

The lack of information on citalopram treatment compliance and duration of use is a limitation of this study. Details on citalopram treatment could provide better insight into the relationship between citalopram use and the development of hyponatremia. Other limitations include the lack of patient clinical data regarding citalopram and thiazide treatment and other concomitant causes of hyponatremia. Changes in thiazide diuretic treatment, such as dose increase, decrease, or cessation, may also impact the occurrence of hyponatremia. Existing research has identified other possible risk factors for hyponatremia on citalopram treatment, such as low body weight and psychosis [19, 23, 24, 27], the data for which were not available for this study. Preexisting conditions or diseases that may independently induce hyponatremia were not identified in our patient population and may skew the results, especially for those patients presenting with a hyponatremic serum baseline value.

Future studies into the risk of hyponatremia with compliant citalopram use, as well as other SSRIs and concurrent use of other diuretic classes, may provide insight into an adverse drug reaction of two, highly prevalent treatments. Particular focus should be given to the risk factors associated with escitalopram use, given its enantiomeric relationship with citalopram. Although not a major finding in this study, quantifying the difference in risks between pharmaceutical manufacturers may be significant in understanding the etiology behind hyponatremia and other adverse drug reactions. Lastly, analysis into the mechanism behind citalopraminduced hyponatremia and the effect of sex on the pathway should be explored, especially since the results of this study contradict existing literature.

\section{Conclusion}

This is the first large-scale, population-based study to explore the incidence and risk factors, based solely on laboratory serum data, for development of hyponatremia in patients initiating citalopram therapy. We report a $16.5 \%$ incidence of hyponatremia after starting citalopram treatment and significant new findings, including a higher incidence in males. The risk factors identified by previous research were largely confirmed in this study, with a notable exception in the effect of sex. Knowledge of the risk factors of hyponatremia following the initiation of citalopram will aid in the prevention of undiagnosed hyponatremia and improve the safety of antidepressant treatment.

\section{Declarations}

Funding This research was funded by a Canadian Institutes of Health Research grant to CN (RN254781-333204).

Conflict of interest None of the authors have any conflicts of interest to disclose.

Ethics approval Ethics approval for this study was obtained from the University of Calgary Conjoint Research Ethics Board (REB 16-2538).

Consent to participate Not applicable.

Consent for publication All authors consent to publication. 
Availability of data and material Because our primary dataset contains potentially identifiable personal information, we do not have research ethics board approval to share the primary dataset.

Code availability Not applicable.

Author contributions AS, CN, ZI, and DS conceived of the study and designed the analysis. AS and CN performed the data analyses. AS wrote the manuscript. AS, CN, ZI, and DS provided critical revisions. AS, CN, ZI, and DS approved the final manuscript.

Open Access This article is licensed under a Creative Commons Attribution-NonCommercial 4.0 International License, which permits any non-commercial use, sharing, adaptation, distribution and reproduction in any medium or format, as long as you give appropriate credit to the original author(s) and the source, provide a link to the Creative Commons licence, and indicate if changes were made. The images or other third party material in this article are included in the article's Creative Commons licence, unless indicated otherwise in a credit line to the material. If material is not included in the article's Creative Commons licence and your intended use is not permitted by statutory regulation or exceeds the permitted use, you will need to obtain permission directly from the copyright holder. To view a copy of this licence, visit http://creativecommons.org/licenses/by-nc/4.0/.

\section{References}

1. Leth-Moller KB, Hansen AH, Torstensson M, Andersen SE, Odum L, Gislasson G, Torp-Pedersen C, Holm EA. Antidepressants and the risk of hyponatremia: a Danish register-based population study. BMJ Open. 2016;6(5):e011200.

2. Leong C, Enns MW, Sareen J, Alessi-Severini S, Bolton J, Prior HJ, Chateau D. New antidepressant use in older adults: a Canadian population-based study (1997-2013). Aging Ment Health. 2016;21(7):720-9.

3. Farmand S, Lindh JD, Calissendorff J, Skov J, Falhammar H, Nathanson D, Mannheimer B. Differences in associations of antidepressants and hospitalization due to hyponatremia. Am J Med. 2018;131(1):56-63.

4. Karkare SU, Bhattacharjee S, Kamble P, Aparasu R. Prevalence and predictors of antidepressant prescribing in nursing home residents in the United States. Am J Geriatr Pharmacother. 2011;9(2):109-19.

5. Phillips JA, Nugent CN. Antidepressant use and method of suicide in the Unites States: variation by age and sex, 1998-2007. Arch Suicide Res. 2013;17(4):360-72.

6. Mars B, Heron J, Kessler D, Davies NM, Martin RM, Thomas $\mathrm{KH}$, Gunnell D. Influences on antidepressant prescribing trends in the UK: 1995-2011. Soc Psychiatry Psychiatr Epidemiol. 2017;52(2):193-200.

7. Sonnenberg CM, Deeg DJ, Comijs HC, van Tilburg W, Beekman AT. Trends in antidepressant use in the older population: results from the LASA-study over a period of 10 years. J Affect Disord. 2008;111(2-3):299-305.

8. Patten SB, Williams JVA, Lavorato DH, Fiest KM, Bulloch AGM, Wang J. Antidepressant use in Canada has stopped increasing. Can J Psychiatry. 2014;59(11):609-14.

9. Solai LK, Mulsant BH, Pollock BG. Selective serotonin reuptake inhibitors for late-life depression: a comparative review. Drugs Aging. 2001;18(5):355-68.
10. Ramamoorthy S, Blakely RD. Phosphorylation and sequestration of serotonin transporters differentially modulated by psychostimulants. Science. 1999;285(5428):763-6.

11. Porsteinsson AP, Drye LT, Pollock BG, Devanand DP, Frangakis $\mathrm{C}$, Ismail Z, Marano C, Meinert C, Meinert CL, Mintzer JE, Munro CA, Pelton G, Rabins PV, Rosenberg PB, Schneider LS, Shade DM, Weintraub D, Yesavage J, Lyketsos CG, CitAD Research Group. Effect of citalopram on agitation in Alzheimer disease: the CitAD randomized clinical trial. JAMA. 2014;311(7):682-91.

12. Hannon MJ, Thompson CJ. The syndrome of inappropriate antidiuretic hormone: prevalence, causes and consequences. Eur J Endocrinol. 2010;162:S5-12.

13. Gandhi S. Risk of hyponatremia in older adults receiving psychotropic drug treatment. Electronic Thesis and Dissertation Repository 2016.

14. Bourgeois JA, Babine SE, Bahadur N. A case of SIADH and hyponatremia associated with citalopram. Psychosomatics. 2002;43(3):241-2.

15. Dirks AC, van Hyfte DM. Recurrent hyponatremia after substitution of citalopram with duloxetine. J Clin Psychopharmacol. 2007;27(3):313.

16. Flores G, Perez-Patrigeon S, Cobos-Ayala C, Vergara J. Severe symptomatic hyponatremia during citalopram therapy-a case report. BMC Nephrol. 2004;5:2.

17. Holland S, Townley S, Summerfield R. Citalopram-a risk factor for postoperative hyponatremia. Anaesthesia. 2003;58(5):491-2.

18. Iraqi A, Baickle E. A case report of hyponatremia with citalopram use. J Am Med Dir Assoc. 2004;5(1):64-5.

19. Mannesse CK, Jansen PA, Van Marum RJ, Sival RC, Kok RM, Haffmans PM, Egberts TC. Characteristics, prevalence, risk factors, and underlying mechanism of hyponatremia in elderly patients treated with antidepressants: a cross-sectional study. Maturitas. 2013;76(4):357-63.

20. Gandhi S, Shariff SZ, Al-Jaishi A, Reiss JP, Mamdani M-M, Hackam DG, Li L, McArthur E, Weir MA, Garg AX. Secondgeneration antidepressants and hyponatremia risk: a population-based cohort study of older adults. Am J Kidney Dis. 2017;69(1):87-96.

21. RStudio Team: RStudio: Integrated Development for R. RStudio, Inc., Boston; 2018. http://www.rstudio.com/.

22. R Core Team: R: A language and environment for statistical computing. R Foundation for Statistical Computing, Vienna; 2018. https://www.R-project.org/.

23. De Picker L, Van Den Eede F, Dumont G, Moorkens G, Sabbe BGC. Antidepressants and the risk of hyponatremia: a class-byclass review of literature. Psychosomatics. 2014;55(6):536-47.

24. Jacob S, Spinler SA. Hyponatremia associated with selective serotonin-reuptake inhibitors in older adults. Ann Pharmacother. 2006;40(9):1618-22.

25. Liu BA, Mittmann N, Knowles SR, Shear NH. Hyponatremia and the syndrome of inappropriate secretion of antidiuretic hormone associated with the use of selective serotonin reuptake inhibitors: a review of spontaneous reports. CMAJ. 1996;155(5):519-27.

26. Strachan J, Shepherd J. Hyponatremia associated with the use of selective serotonin re-uptake inhibitors. Aust N Z J Psychiatry. 1998;32(2):295-8.

27. Wee R, Lim WK. Selective serotonin re-uptake inhibitors (SSRIs) and hyponatremia in the elderly. Int J Geriatr Psychiatry. 2004;19(6):590-1.

28. Greenblatt HK, Greenblatt DJ. Antidepressant-associated hyponatremia in the elderly. J Clin Psychopharmacol. 2016;36(6):545-9.

29. Coupland C, Dhiman P, Morriss R, Arthur A, Barton G, HippisleyCox J. Antidepressant use and risk of adverse outcomes in older people: population based cohort study. BMJ. 2011;343:d4551. 
30. Bowen PD. Use of selective serotonin reuptake inhibitors in the treatment of depression in older adults: identifying and managing potential risk for hyponatremia. Geriatr Nurs. 2009;30(2):85-9.

31. Filippatos TD, Makri A, Elisaf MS, Liamis G. Hyponatremia in the elderly: challenges and solutions. Clin Interv Aging. 2017;12:1957-65.

32. Bissram M, Scott FD. Risk factors for symptomatic hyponatremia: the role of pre- existing asymptomatic hyponatremia. Intern Med J. 2007;37(3):149-55.

33. Nagler EV, Vanmassenhove J, Van der Veer SN, Nistor I, Van Biesen W, Webster AC, Vanholder R. Diagnosis and treatment of hyponatremia: a systematic review of clinical practice guidelines and consensus statements. BMC Med. 2014;12:1.

34. Burst V, Grundmann F, Kubacki T, Greenberg A, Becker I, Rudolf D, Verbalis J. Thiazide-associated hyponatremia, report of the hyponatremia registry: an observational multicenter international study. Am J Nephrol. 2017;45(5):420-30.

35. Rosner MH. Severe hyponatremia associated with the combined use of thiazide diuretics and selective serotonin reuptake inhibitors. Am J Med Sci. 2004;327(2):109-11.

36. Voyer P, Cohen D, Lauzon S, Collin J. Factors associated with psychotropic drug use among community-dwelling older persons: a review of empirical studies. BMC Nurs. 2004;3(1):3.

37. Weissman MM, Bland R, Joyce PR, Newman S, Wells JE, Wittchen H-U. Sex differences in rates of depression: crossnational perspectives. J Affect Disord. 1993;29(2-3):77-84.

38. Sun H, Sun M. Age- and gender- dependent associations of blood pressure and serum sodium and potassium - renal and extrarenal regulations. J Am Soc Hypertens. 2018;12(5):392-401.

39. Veiras LC, Girardi ACC, Curry J, Pei L, Ralph DL, Tran A, Castelo-Branco RC, Pastor-Soler N, Arranz CT, Yu ASL, McDonough AA. Sexual dimorphism pattern of renal transporters and electrolyte homeostasis. JASN. 2017;28(12):3504-17.

40. Jovanovic H, Lundberg J, Karlsson P, Cerin A, Saijo T, Varrone A, Halldin C, Nordström AL. Sex differences in the serotonin 1A receptor and serotonin transporter binding in the human brain measured by PET. Neuroimage. 2008;39(3):1408-19.
41. Dunne S, Shannon B, Dunna C, Cullen W. A review of the differences and similarities between generic drugs and their original counterparts, including economic benefits associated with usage of generic medicines, using Ireland as a case study. BMC Pharmacol Toxicol. 2013;14:1.

42. Bech R, Tanghøj P, Andersen HF, Overø K. Citalopram doseresponse revisited using an alternative psychometric approach to evaluate clinical effects of four fixed citalopram doses compared to placebo in patients with major depression. Psychopharmacology. 2002;163(1):20-5.

43. Gupta M, Neavin D, Liu D, Biernacka J, Hall-Flavin D, Bobo WV, Frye MA, Skime M, Jenkins GD, Batzler A, Kalari K, Matson W, Bhasin SS, Zhu H, Mushiroda T, Nakamura Y, Kubo M, Wang L, Kaddurah-Daouk R, Weinshilboum RM. TSPAN5, ERICH3 and selective serotonin reuptake inhibitors in major depressive disorder: pharmacometabolomics-informed pharmacogenomics. Mol Psychiatry. 2016;21(12):1717-25.

44. Ismail Z, Granger R, Pollock BG. General principles of pharmacologic therapy. In: Tasman A, Kay J, Lieberman JA, First MB, Riba MB, editors. Psychiatry. West Sussex: Academic; 2015. p. 2025-40.

45. Kandalam V, Lau CK, Guo M, Ma I, Naugler C. Inappropriate repeat testing of complete blood count (CBC) and electrolyte panels in inpatients from Alberta, Canada. Clin Biochem. 2020;77:32-5.

46. Barber J, Guo M, Nguyen LT, Thomas R, Turin TC, Vaska M, Naugler C, COAPT (Collaboration On Assessing Physician Testing). Sociodemographic correlated of clinical laboratory test expenditures in a major Canadian city. Am J Clin Pathol. 2017;148(1):91-6.

47. Fischer SH, Field TS, Gagne SJ, Mazor KM, Preusse P, Reed G, Peterson D, Gurwitz JH, Tjia J. Patient completion of laboratory tests to monitor medication therapy: a mixed-methods study. J Gen Intern Med. 2013;28(4):513-21.

48. McAlister FA, Tu K, Majumdar SR, Padwal R, Chen Z, Campbell NR. Laboratory testing in newly treated elderly hypertensive patients without co-morbidities: a population-based cohort study. Open Med. 2007;1(2):e60-67.

\section{Authors and Affiliations}

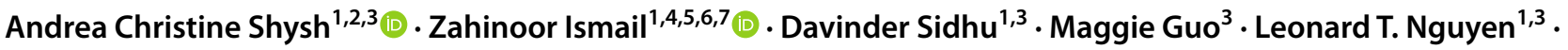 Christopher Naugler ${ }^{1,2,3,5}$}

1 Department of Pathology and Laboratory Medicine, Cumming School of Medicine, University of Calgary, Calgary, Canada

2 Department of Family Medicine, Cumming School of Medicine, University of Calgary, Calgary, Canada

3 Alberta Precision Laboratories (Formerly Calgary Laboratory Services), Calgary, Canada

4 Hotchkiss Brain Institute, University of Calgary, Calgary, Canada
5 Department of Community Health Sciences, Cumming School of Medicine, University of Calgary, Calgary, Canada

6 Department of Psychiatry, Cumming School of Medicine, University of Calgary, Calgary, Canada

7 Department of Clinical Neurosciences, Cumming School of Medicine, University of Calgary, Calgary, Canada 